\title{
Letter to the Editor concerning "Nucleus pulposus deformation in response to lumbar spine lateral flexion: an in vivo MRI investigation" by Fazey PJ et al. (2010) Eur Spine J 19:1115-1120
}

\author{
Edwin C. W. Lim
}

Received: 18 July 2010/Accepted: 22 August 2010/Published online: 4 September 2010

(C) Springer-Verlag 2010

Dear Editor,

With interest I read the article by Fazey et al. [1] describing a meticulously conducted study in which they used an image analysis software (NIH Image-J, Bethesda, USA) to investigate hydration pattern within the lumbar intervertebral disc (IVD) in response to lateral flexion. I want to commend on their effort in seeking to share this information through publication of their work. Such novel method employed the use of linear pixel samples across the width of the disc from T2-weighted magnetic resonance imaging (MRI) images in order to determine the magnitude and direction of the displacement of the nucleus pulposus (NP) in lumbar IVD.

With these, I do have a question regarding such technique which was validated previously [2]. The authors mentioned that three lines were placed across the mid-disc region from right to left in the Methods on p. 1117. However, they did not specify the distance between these lines. I believe that an interval of $0.5 \mathrm{~cm}$ compared to $1 \mathrm{~cm}$ between the lines may potentially yield different hydration profile of the disc. Furthermore, what should the optimal interval between the lines be in order to yield the best hydration profile of the disc? On another note, it is plausible that assessment of the hydration profile would be improved if the number of lines (linear pixel samples) across the width of the disc is increased. Nonetheless, with reference to the Discussion p. 1120, I am also in agreement with the authors that a more complex 3-D profile maps of the annulus and NP would provide an improved evaluation of hydration patterns across the entire IVD via visual inspection.

I also believe that such important detail may have been overlooked during the preparation of the manuscript. But I realised that it was also not reported in other publications of their work as well $[2,3]$. As such, this point is highlighted in the interest of other readers or researchers who may be interested to replicate the study in the near future.

\section{References}

1. Fazey PJ, Takasaki H, Singer KP (2010) Nucleus pulposus deformation in response to lumbar spine lateral flexion: an in vivo MRI investigation. Eur Spine J 19:1115-1120

2. Fazey PJ, Song S, Monsas S et al (2006) An MRI investigation of intervertebral disc deformation in response to torsion. Clin Biomech (Bristol, Avon) 21:538-542

3. Takasaki H, May S, Fazey PJ, Hall T (2010) Nucleus pulposus deformation following the McKenzie concept: a single case report with in vivo MRI investigation. J Man Manip Ther (in press)
E. C. W. Lim $(\varangle)$

Department of Physiotherapy, Singapore General Hospital, Outram Road, Singapore 169608, Singapore

e-mail: edwin.lim.c.w@sgh.com.sg 